\title{
Integrability for nonlinear time-delay systems
}

\author{
Arvo Kaldmäe, Claudia Califano Member, IEEE and Claude H. Moog, Fellow, IEEE
}

\begin{abstract}
In this paper the notion of integrability is defined for 1-forms defined in the time-delay context. While in the delay-free case, a set of 1-forms defines a vector space, it is shown that 1-forms computed for time-delay systems have to be viewed as elements of a module over a certain non-commutative polynomial ring. Two notions of integrability are defined, strong and weak integrability, which coincide in the delay-free case. Necessary and sufficient conditions are given to check if a set of 1 -forms is strongly or weakly integrable. To show the importance of the topic, integrability of 1-forms is used to characterize the accessibility property for nonlinear time-delay systems. The possibility of transforming a system into a certain normal form is also considered.
\end{abstract} bility

Index Terms - Time-delay systems, algebraic methods, accessi-

\section{INTRODUCTION}

Time-delay systems are used in many important areas, like telecommunications, remote control and biological systems (see [1] and the references therein). The great success of algebraic [7] and differential geometric [6], [8] methods for delay-free systems has motivated many authors to generalize the approaches to the time-delay case [2], [4], [9], [10], [11], [12], [13], [16]. Of major importance in these approaches is the notion of integrability of codistributions (or distributions). In the delay-free case, the integrability is fully characterized by the so-called Frobenius Theorem. The class of time-delay systems is a special class of infinite dimensional systems though, it was shown in [4] that Frobenius Theorem is still valid to derive specific results. In [5] and [2], integrability was tackled in the case of one-dimensional submodules and a necessary and sufficient condition was derived. A sufficient condition for the general case was also given in [2]. A different approach was used in [4], where the integrability was characterized using the extended Lie brackets.

At this point, there is no general theory about integrability of 1-forms/codistributions in the case of time-delay systems. The main goal in this paper is to clarify those notions of integrability of 1 -forms and which are not fully captured by the integrability of vector fields. In [2], the existence of an exact basis is defined for a module, while in [5] as the existence of an exact basis is defined for the closure of a module.

A. Kaldmäe is with Institute of Cybernetics at TUT, Akadeemia tee 21, 12618, Tallinn, Estonia and LUNAM, IRCCyN, UMR CNRS 6597, 1 rue de la Noë, BP 92101, 44321 Nantes Cedex 3, France e-mail: (arvo@cc.ioc.ee).

C. Califano is with Dipartimento di Ingegneria Informatica Automatica e Gestionale Antonio Ruberti, Università di Roma La Sapienza, Via Ariosto 25, 00185, Italy e-mail: (claudia.califano@uniroma1.it).

C.H. Moog is with LUNAM, IRCCyN, UMR CNRS 6597, 1 rue de la Noë, BP 92101, 44321 Nantes Cedex 3, France e-mail: (Claude.Moog @irccyn.ecnantes.fr).

Manuscript received xxxx, 20xx; revised xxxx, 20xx. The work of A. Kaldmäe was supported by the Estonian Research Council, personal research funding grant PUT481 and by the Estonian Doctoral School in Information and Communication Technology.
In this paper, we use the notion of closure of a submodule [3] to define two notions of integrability - strong integrability and weak integrability - and give necessary and sufficient conditions to check both these properties for a set of 1forms. The relationship between the obtained results and the dual results of [4] is also discussed. Then, two problems are considered, where the integrability of 1 -forms plays a key role. Accessibility of nonlinear time-delay systems is characterized through the integrability of a certain submodule and conditions are found under which a given system can be transformed into a certain normal form. Preliminary results and examples can be found in [17].

The paper is organized in the following manner. In Section II, basic mathematical notions are given, which will be used in the paper. In Section III, the main results are presented. The integrability of 1-forms is defined and the condition is given, together with two algorithms, to check integrability. In section IV, the connection between the results of section III and [4] is argued. Applications of integrability of 1-forms are considered in Section V. The paper ends with some conclusions.

\section{PRELIMINARIES}

Non-commutative algebra is used to define the integrability of 1-forms and to find the necessary and sufficient conditions to get integrability (which is done in Section III). More precisely, the proposed method refers to modules over noncommutative rings (see [5], [9]). In this section, the mathematics and definitions beyond this method are introduced.

Let $\mathcal{K}$ denote the field of meromorphic functions that depend on a finite number of variables from the set $\{x(t-i) ; i \in \mathbb{N}\}$, $\operatorname{dim}(x(t))=n$. Also, denote by $\mathcal{E}$ the vector space spanned by the differentials $\{\mathrm{d} x(t-i) ; i \in \mathbb{N}\}$ over the field $\mathcal{K}$. The elements of $\mathcal{E}$ are called 1-forms.

Consider the time shift acting over functions $\delta: \mathcal{K} \rightarrow \mathcal{K}$ defined as $\delta f(x(t-i) ; i \in \mathbb{N}):=f(x(t-i-1) ; i \in \mathbb{N})$. On the 1-form $\omega=\sum_{i=1}^{n} \sum_{j=0}^{k} a_{i} \mathrm{~d} x_{i}(t-j)$, one gets that the time shift $\delta \omega$ of $\omega$ is given by

$$
\delta \omega=: \omega^{-}=\sum_{i=1}^{n} \sum_{j=0}^{k} \delta\left(a_{i}\right) \mathrm{d} x_{i}(t-j-1) .
$$

Accordingly, $\omega^{-p}:=\delta \omega^{-p+1}$. Furthermore, $\omega$ is said to be exact if there exists $\varphi \in \mathcal{K}$ such that $\omega=\mathrm{d} \varphi$. The use of exterior differentiation and of the wedge product allows to state in a concise manner both Poincaré Lemma and Frobenius Theorem [14]:

- the 1 -form $\omega$ is locally exact if and only if $\mathrm{d} \omega=0$;

- the codistribution $\operatorname{span}_{\mathcal{K}}\left\{\omega_{1}, \ldots, \omega_{q}\right\}$ is integrable if and only if the $q+2$-forms $\mathrm{d} \omega_{i} \wedge \omega_{1} \wedge \ldots \wedge \omega_{q}$ are zero for $i=1, \ldots, q$, where $\wedge$ denotes the wedge product of differential forms [14]. 
The following notation is also used:

$$
\mathrm{d} \omega=0 \bmod \operatorname{span}_{\mathcal{K}}\left\{\bar{\omega}_{1}, \ldots, \bar{\omega}_{q}\right\}
$$

means that $\mathrm{d} \omega \wedge \bar{\omega}_{1} \wedge \cdots \wedge \bar{\omega}_{q}=0$.

Next, the non-commutative ring of polynomials $\mathcal{K}(\vartheta]$ is constructed. The elements of this ring are polynomials in the form $a_{0}+a_{1} \vartheta+\cdots+a_{s} \vartheta^{s}$ for some finite $s \in \mathbb{N}$ and $a_{i} \in \mathcal{K}$, $i=0, \ldots, s$. Addition is defined on this ring as usual, but the rule for multiplication is $\vartheta \psi=\delta(\psi) \vartheta$ for some $\psi \in \mathcal{K}$. Similarly, $\vartheta(\omega)=\delta \omega$, and when no confusion arises, $\vartheta(\omega)$ will be denoted $\vartheta \omega$. The set of matrices $\mathcal{K}(\vartheta]^{k \times s}$ is also used in this paper.

Definition 1: [15] A matrix $A(\vartheta) \in \mathcal{K}(\vartheta]^{k \times k}$ is unimodular if it is invertible within the ring of polynomial matrices, i.e. if there exists a $B(\vartheta) \in \mathcal{K}(\vartheta]^{k \times k}$ such that $A(\vartheta) B(\vartheta)=$ $B(\vartheta) A(\vartheta)=I_{k}$.

Example 1: The matrix

$$
A(\vartheta)=\left(\begin{array}{cc}
1 & x_{2}(t-1) \vartheta \\
\vartheta & 1+x_{2}(t-2) \vartheta^{2}
\end{array}\right)
$$

is unimodular, since the matrix

$$
A(\vartheta)^{-1}=\left(\begin{array}{cc}
1+x_{2}(t-1) \vartheta^{2} & -x_{2}(t-1) \vartheta \\
-\vartheta & 1
\end{array}\right)
$$

is such that $A(\vartheta) A(\vartheta)^{-1}=A(\vartheta)^{-1} A(\vartheta)=I_{2}$. Note that while any unimodular matrix has full rank, the converse is not true. For example, there is no polynomial inverse for $(1+\vartheta)$.

Let us now note that the set of 1 -forms $\mathcal{E}$ has the structure of a vector space over the field $\mathcal{K}$. However, it has also the structure of a module, denoted $\mathcal{M}$, over the $\operatorname{ring} \mathcal{K}(\vartheta]$, i.e.

$$
\mathcal{M}=\operatorname{span}_{\mathcal{K}(\vartheta]}\{\mathrm{d} x(t)\} .
$$

Example 2: The 1-forms $\mathrm{d} x_{1}(t)$ and $\mathrm{d} x_{1}(t-1)$ are independent over the field $\mathcal{K}$, but dependent over the ring $\mathcal{K}(\vartheta]$, since $\vartheta \mathrm{d} x_{1}(t)-\mathrm{d} x_{1}(t-1)=0$. This simple example shows that the action of time-delay is taken into account in $\mathcal{M}$, but not in $\mathcal{E}$. This motivates the definition of the module $\mathcal{M}$.

A left-submodule of $\mathcal{M}$ consists of all possible linear combinations of given 1-forms (or row-vectors) $\left\{\omega_{1}, \ldots, \omega_{k}\right\}$ over the ring $\mathcal{K}(\vartheta]$, i.e. linear combinations of row-vectors. A left-submodule, generated by $\left\{\omega_{1}, \ldots, \omega_{k}\right\}$, is denoted by $\mathcal{A}=\operatorname{span}_{\mathcal{K}(\vartheta]}\left\{\omega_{1}, \ldots, \omega_{k}\right\}$. A right-submodule of $\hat{\mathcal{M}}$ [4] consists of all possible linear combinations of columnvectors $q_{1}, \ldots, q_{l}, q_{i} \in \mathcal{K}(\vartheta]^{n \times 1}$, and is denoted by $\Delta=$ $\operatorname{span}_{\mathcal{K}(\vartheta]}\left\{q_{1}, \ldots, q_{l}\right\}$.

Definition 2: The left closure of a left-submodule $\mathcal{A}$ of $\mathcal{M}$, denoted by $c l_{\mathcal{K}(\vartheta]}(\mathcal{A})$, is defined as $c l_{\mathcal{K}(\vartheta]}(\mathcal{A})=\{\omega \in \mathcal{M} \mid$ $\exists p(\vartheta) \in \mathcal{K}(\vartheta], p(\vartheta) \omega \in \mathcal{A}\}$.

By definition, the left closure of the left-submodule $\mathcal{A}$ is the largest left-submodule, containing $\mathcal{A}$, with the same rank as $\mathcal{A}$.

Definition 3: The right closure of a right-submodule $\Delta$ of $\hat{\mathcal{M}}$, denoted by $\operatorname{cl}_{\mathcal{K}(\vartheta]}(\Delta)$, is defined as $c l_{\mathcal{K}(\vartheta]}(\Delta)=\{X \in$ $\hat{\mathcal{M}} \mid \exists q(\vartheta) \in \mathcal{K}(\vartheta], X q(\vartheta) \in \Delta\}$.

The right closure of the right-submodule $\Delta$ is the largest rightsubmodule, containing $\Delta$, with the same rank as $\Delta$.

Consider a left-submodule $\mathcal{A}$ of $\mathcal{M}$ and let the 1 -forms $\omega$ be the basis of $\mathcal{A}$. These 1 -forms can be written as $\omega=$ $P(\vartheta) \mathrm{d} x(t)$ for some matrix $P(\vartheta) \in \mathcal{K}(\vartheta]^{k \times n}$.
Definition 4: The right-kernel (right-annihilator) of the leftsubmodule $\mathcal{A}$ is the right-submodule $\Delta$ containing all vectors $q(\vartheta) \in \hat{\mathcal{M}}$ such that $P(\vartheta) q(\vartheta)=0$.

From Definition 4, the right-kernel is necessarily closed. Consider a right-submodule $\Delta=\operatorname{span}_{\mathcal{K}(\vartheta]}\left\{q_{1}(\vartheta), \ldots, q_{l}(\vartheta)\right\}$.

Definition 5: The left-kernel (left-annihilator) of $\Delta$ is the left-submodule $\mathcal{A}$ containing all 1-forms $\omega(\vartheta) \in \mathcal{M}$ such that $\omega(\vartheta) \Delta=0$.

Again, from Definition 5, the left-kernel is necessarily closed. Finally, it is straightforward to prove the following.

Lemma 1: The right-kernels of the left-submodules $\mathcal{A}$ and $c l_{\mathcal{K}(\vartheta]}(\mathcal{A})$ are equal. The left-kernels of the right-submodules $\Delta$ and $\operatorname{cl}_{\mathcal{K}(\vartheta]}(\Delta)$ are equal.

\section{RESUlTS ON INTEGRABILITY OF 1-FORMS}

In the present section a set of 1 -forms $\left\{\omega_{1}, \ldots, \omega_{k}\right\}$ independent over $\mathcal{K}(\vartheta]$ is considered (that is, there is no non zero linear combination over the ring $\mathcal{K}(\vartheta]$ which vanishes). As it will be shown hereafter, the fact of considering 1-forms as elements of $\mathcal{M}$ naturally leads to two different notions of integrability. If 1 -forms are considered as elements of vector space $\mathcal{E}$, there is only one single notion of integrability.

In fact, as it happens in the delay-free case, if the set of 1forms $\left\{\omega_{1} \ldots, \omega_{k}\right\}$ are considered over $\mathcal{K}$, then they are said to be integrable if there exists an invertible matrix $A \in \mathcal{K}^{k \times k}$ and functions $\varphi=\left(\varphi_{1}, \ldots, \varphi_{k}\right)^{T}$, such that $\omega=A \mathrm{~d} \varphi$. The full rank of $A$ guarantees the invertibility of $A$, since $\mathcal{K}$ is a field. Instead, if the 1 -forms $\left\{\omega_{1} \ldots, \omega_{k}\right\}$ are viewed as elements of the module $\mathcal{M}$, then the matrix $A \in \mathcal{K}(\vartheta]^{k \times k}$ instead of $\mathcal{K}^{k \times k}$. Since $A(\vartheta)$ may be of full rank but not unimodular, it is necessary to distinguish two cases. Accordingly, one has the following two definitions of integrability.

Definition 6: A set of $k 1$-forms $\left\{\omega_{1}, \ldots, \omega_{k}\right\}$, independent over $\mathcal{K}(\vartheta]$, is said to be strongly integrable if there exist $k$ independent functions $\left\{\varphi_{1}, \ldots, \varphi_{k}\right\}$, such that

$$
\operatorname{span}_{\mathcal{K}(\vartheta]}\left\{\omega_{1}, \ldots, \omega_{k}\right\}=\operatorname{span}_{\mathcal{K}(\vartheta]}\left\{\mathrm{d} \varphi_{1}, \ldots, \mathrm{d} \varphi_{k}\right\} .
$$

A set of $k 1$-forms $\left\{\omega_{1}, \ldots, \omega_{k}\right\}$, independent over $\mathcal{K}(\vartheta]$, is said to be weakly integrable if there exist $k$ independent functions $\left\{\varphi_{1}, \ldots, \varphi_{k}\right\}$, such that

$$
\operatorname{span}_{\mathcal{K}(\vartheta]}\left\{\omega_{1}, \ldots, \omega_{k}\right\} \subseteq \operatorname{span}_{\mathcal{K}(\vartheta]}\left\{\mathrm{d} \varphi_{1}, \ldots, \mathrm{d} \varphi_{k}\right\}
$$

If the 1-forms $\omega=\left(\omega_{1}, \cdots, \omega_{k}\right)^{T}$ are strongly (respectively weakly) integrable, then the left-submodule $\operatorname{span}_{\mathcal{K}(\vartheta]}\left\{\omega_{1}, \ldots, \omega_{k}\right\}$ is said to be strongly (respectively weakly) integrable.

Clearly, strong integrability yields weak integrability. Also, the 1-forms $\omega$ are weakly integrable if and only if there exists a matrix $A(\vartheta) \in \mathcal{K}(\vartheta]^{k \times k}$ with full rank and functions $\varphi=$ $\left(\varphi_{1}, \ldots, \varphi_{k}\right)^{T}$ such that $\omega=A(\vartheta) \mathrm{d} \varphi$. If in addition the matrix $A(\vartheta)$ can be chosen to be unimodular, then the 1-forms $\omega$ are also strongly integrable.

Example 3: The 1-form $\omega_{1}=\mathrm{d} x(t)+x(t-1) \mathrm{d} x(t-1)$ is weakly integrable since $\omega_{1}=(1+x(t-1) \vartheta) \mathrm{d} x(t)$. It is also strongly integrable as $\omega_{1}=\mathrm{d}\left(x(t)+1 / 2 x(t-1)^{2}\right)$. Instead, the 1-form $\omega_{2}=\mathrm{d} x_{1}(t)+x_{2}(t) \mathrm{d} x_{1}(t-1)=\left(1+x_{2}(t) \vartheta\right) \mathrm{d} x_{1}(t)$ 
is weakly integrable, but not strongly integrable, because the polynomial $1+x_{2}(t) \vartheta$ is not invertible.

Remark 1: Note that integrability of a closed left-submodule $\operatorname{span}_{\mathcal{K}(\vartheta]}\left\{\omega_{1}, \ldots, \omega_{k}\right\}$ always implies strong integrability. As a consequence, the two notions of strong and weak integrability coincide in case of delay-free 1-forms.

Integrability of a set of $k 1$-forms $\left\{\omega_{1}, \ldots, \omega_{k}\right\}$ is tested thanks to the so-called

Derived Flag Algorithm (DFA):

Starting from a given $I_{0}$ the algorithm computes

$$
I_{i}=\operatorname{span}_{\mathcal{K}}\left\{\omega \in I_{i-1} \mid \mathrm{d} \omega=0 \bmod I_{i-1}\right\} .
$$

The sequence (1) converges as it defines a strictly decreasing sequence of vector spaces $I_{i}$ and by the standard Frobenius Theorem, the limit $I_{\infty}$ has an exact basis, which represents the largest integrable codistribution contained in $I_{0}$.

In order to define $I_{0}$ one has to note that when considering a set of $k 1$-forms $\left\{\omega_{1}, \cdots, \omega_{k}\right\}$, some shifts of $\omega_{i}$ are required for integration. It follows that the inizialization

$$
I_{0}^{p}=\operatorname{span}_{\mathcal{K}}\left\{\omega_{1}, \ldots, \omega_{k}, \ldots, \omega_{1}^{-p}, \ldots, \omega_{k}^{-p}\right\},
$$

allows to compute the smallest number of time shifts required for the given 1-forms for the maximal integration of the submodule. More precisely, the sequence $I_{i}^{p}$ defined by (1) converges to an integrable vector space

$$
I_{\infty}^{p}=\operatorname{span}_{\mathcal{K}}\left\{\mathrm{d} \varphi_{1}^{p}, \ldots, \mathrm{d} \varphi_{\gamma_{p}}^{p}\right\}
$$

for some $\gamma_{p} \geq 0$. By definition, $\mathrm{d} \varphi_{i}^{p} \in \operatorname{span}_{\mathcal{K}(\vartheta]}\left\{\omega_{1}, \ldots, \omega_{k}\right\}$ for $i=1, \ldots, \gamma_{p}$ and $p \geq 0$. The exact 1 -forms $\mathrm{d} \varphi_{i}^{p}, i=$ $1, \ldots, \gamma_{p}$, are independent over $\mathcal{K}$, but may not be independent over $\mathcal{K}(\vartheta]$. A basis for $\operatorname{span}_{\mathcal{K}(\vartheta]}\left\{\mathrm{d} \varphi_{1}^{p}, \ldots, \mathrm{d} \varphi_{\gamma_{p}}^{p}\right\}$ is obtained by computing a basis for

$$
I_{\infty}^{0} \cup \bigcup_{i=1}^{p}\left[I_{\infty}^{i} \bmod \left(I_{\infty}^{i-1}, \delta I_{\infty}^{i-1}\right)\right]
$$

as $I_{\infty}^{i}+\delta I_{\infty}^{i} \subset I_{\infty}^{i+1}$.

Remark 2: A different initialization of derived flag algorithm is

$$
\begin{aligned}
\tilde{I}_{0}^{p}= & \operatorname{span}_{\mathcal{K}}\left\{\operatorname{span}_{\mathcal{K}(\vartheta]}\left\{\omega_{1}, \cdots, \omega_{k}\right\} \cap\right. \\
& \left.\operatorname{span}_{\mathcal{K}}\{\mathrm{d} x(t), \cdots, \mathrm{d} x(t-p)\}\right\} .
\end{aligned}
$$

which allows to compute for each $p \geq 0$, the exact differentials contained in the given submodule and which depend on $x(t), \ldots, x(t-p)$ only. Both initialization allow the algorithm to converge towards the same integrable submodule over $\mathcal{K}(\vartheta]$, but follow different steps, as shown in the next example.

Example 4: Let $\operatorname{span}_{\mathcal{K}(\vartheta]}\{\mathrm{d} x(t-2)\}$. On one hand, initialization (2) is completed for $p=0$ as no time-shift of $\mathrm{d} x(t-2)$ is required for its integration. On the other hand, initialization (4) yields a 0 limit for $p=0$ and $p=1$ as the exact differential involves larger delays than $x(t)$ and $x(t-1)$. The final result is obtained for $p=2$.

Assume that the maximum delay that appears in $\left\{\omega_{1}, \ldots, \omega_{k}\right\}$ (either in the coefficients or differentials) is $s$. The necessary and sufficient condition for strong integrability of 1 -forms $\left\{\omega_{1}, \ldots, \omega_{k}\right\}$ is given by the following theorem in terms of the limit $I_{\infty}^{p}$.

Theorem 1: A set of 1-forms $\left\{\omega_{1}, \ldots, \omega_{k}\right\}$, independent over $\mathcal{K}(\vartheta]$, is strongly integrable if and only if there exists an index $p \leq s(k-1)$ such that starting from $I_{0}^{p}$ defined by (2), the derived flag algorithm (1) converges to $I_{\infty}^{p}$ given by (3) with

$$
\omega_{i} \in \operatorname{span}_{\mathcal{K}(\vartheta]}\left\{\mathrm{d} \varphi_{1}^{p}, \ldots, \mathrm{d} \varphi_{\gamma_{p}}^{p}\right\}
$$

for $i=1, \ldots, k$.

Proof. Necessity. If a set of 1-forms $\left\{\omega_{1}, \ldots, \omega_{k}\right\}$, independent over $\mathcal{K}(\vartheta]$, is strongly integrable, then there exist $k$ functions $\varphi_{i}, i=1, \ldots, k$, such that $\operatorname{span}_{\mathcal{K}(\vartheta]}\left\{\omega_{1}, \ldots, \omega_{k}\right\}=\operatorname{span}_{\mathcal{K}(\vartheta]}\left\{\mathrm{d} \varphi_{1}, \ldots, \mathrm{d} \varphi_{k}\right\}$. Thus $\omega_{i} \in \operatorname{span}_{\mathcal{K}(\vartheta]}\left\{\mathrm{d} \varphi_{1}, \ldots, \mathrm{d} \varphi_{k}\right\}$ and $\mathrm{d} \varphi_{i} \in$ $\operatorname{span}_{\mathcal{K}}\left\{\omega_{1}, \ldots, \omega_{k}, \ldots, \omega_{1}^{-p}, \ldots, \omega_{k}^{-p}\right\}$ for $i=1, \ldots, k$ and some $p \geq 0$. Clearly, $\mathrm{d} \varphi_{i} \in I_{\infty}^{p}$ and the condition (5) is satisfied.

It remains to show that $p \leq s(k-1)$. Note that there exist infinitely many pairs $(A(\vartheta), \varphi)$, that satisfy $\omega=A(\vartheta) \mathrm{d} \varphi$. Since the degree of unimodular matrices $A(\vartheta)$ has a lower bound, then one can find a pair $(A(\vartheta), \varphi)$, where the degree of matrix $A(\vartheta)$ is minimal among all possible pairs. Let $A(\vartheta)$ be such a unimodular matrix for some functions $\varphi=\left(\varphi_{1}, \ldots, \varphi_{k}\right)^{T}$. Note that $A(\vartheta)$ and $\varphi$ are not unique.

We show that the degree of $A(\vartheta)$ is less or equal to $s$. By contradiction, assume that the degree of $A(\vartheta)$ is larger than $s$, for example $s+1$. Then for some $i$

$$
\omega_{i}=a_{1}^{i}(\vartheta) \mathrm{d} \varphi_{1}+\cdots+a_{k}^{i}(\vartheta) \mathrm{d} \varphi_{k}
$$

where $a_{j}^{i}(\vartheta) \in \mathcal{K}(\vartheta], j=1, \ldots, k$, and at least one polynomial $a_{j}^{i}(\vartheta)$ has degree $s+1$.

Let $a_{j}^{i}(\vartheta)=\sum_{l=0}^{s+1} a_{j, l}^{i} \vartheta^{l}, j=1, \ldots, k$. From (6) one gets

$$
\omega_{i}=\sum_{j=1}^{k} \sum_{\ell=0}^{s+1} a_{j, \ell}^{i} \mathrm{~d} \varphi_{j}^{-i},
$$

where at least one coefficient $a_{j, s+1}^{i} \in \mathcal{K}$ is non-zero. For simplicity assume that $a_{1, s+1}^{i} \neq 0$ and $a_{\gamma, s+1}^{i}=0$ for $\gamma=$ $2, \ldots, k$. We have assumed that the maximum delay in $\omega_{i}$ is $s$, but the maximum delay in $\mathrm{d} \varphi_{1}^{-s-1}$ is at least $s+1$.

Note that $\mathrm{d} \varphi_{1}, \ldots, \mathrm{d} \varphi_{1}^{-s-1}, \ldots, \mathrm{d} \varphi_{k}^{-s-1}$ are independent over $\mathcal{K}$. Therefore, to eliminate $\mathrm{d} \varphi_{1}^{-s-1}$ from (7),

$$
\mathrm{d} \varphi_{1}^{-s-1}=\sum_{j=1}^{k} b_{j}(\vartheta) \mathrm{d} \varphi_{j}+\bar{\omega}
$$

for some coefficients $b_{j}(\vartheta) \in \mathcal{K}(\vartheta]$. Let $l_{j}=\operatorname{deg}_{j}(\vartheta) \leq s$ and the 1 -form $\bar{\omega} \in \operatorname{span}_{\mathcal{K}}\left\{\mathrm{d} x, \mathrm{~d} x^{-}, \ldots, \mathrm{d} x^{-s}\right\}$. Let $l:=$ $\min \left\{l_{j}\right\}$. For clarity, let $l=l_{2}$ and $b_{2}(\vartheta)=\vartheta^{l}$. We show that $\bar{\omega}$ can be chosen such that it is integrable. By contradiction, assume that $\bar{\omega}$ can not be chosen integrable. Then, the coefficients of $\bar{\omega}$ must depend on higher delays than $s$. Since $\bar{\omega}$ is not integrable, then the coefficients of $a_{1, s+1}^{i} \bar{\omega}$ depend also on higher delays than $s$. Now, substitute $a_{1, s+1}^{i} \mathrm{~d} \varphi_{1}^{-s-1}$ to (7). One gets that $\omega_{i}$ depends on $a_{1, s+1}^{i} \bar{\omega}$ and thus also on higher delays than $s$. This is a contradiction and thus $\bar{\omega}$ can be chosen integrable. 
Let $\bar{\omega}=a \mathrm{~d} \phi^{-l}$ for some $a, \phi \in \mathcal{K}$. Then $\operatorname{span}_{\mathcal{K}(\vartheta]}\left\{\mathrm{d} \varphi_{1}, \cdots, \mathrm{d} \varphi_{k}\right\}=\operatorname{span}_{\mathcal{K}(\vartheta]}\left\{\mathrm{d} \varphi_{1}, \mathrm{~d} \phi, \mathrm{d} \varphi_{3}, \cdots, \mathrm{d} \varphi_{k}\right\}$ and there exists an unimodular matrix $\bar{A}(\vartheta)$ with smaller degree than $A(\vartheta)$, and functions $\bar{\varphi}=\left(\varphi_{1}, \phi, \varphi_{3}, \ldots, \varphi_{k}\right)^{T}$ such that $\omega=\bar{A}(\vartheta) \mathrm{d} \bar{\varphi}$, which leads to a contradiction. Thus the degree of $A(\vartheta)$ must be less than or equal to $s$ and the degree of $A^{-1}(\vartheta)$ is less or equal to $s(k-1)$, i.e. $p \leq s(k-1)$. The general case requires a more technical proof.

Sufficiency. Let $I_{\infty}^{p}=\operatorname{span}_{\mathcal{K}}\{\mathrm{d} \varphi\}$, where $p \leq s(k-$ 1). By construction $I_{\infty}^{p} \subset \operatorname{span}_{\mathcal{K}(\vartheta]}\left\{\omega_{1}, \ldots, \omega_{k}\right\}$ and by (5) $\omega_{i} \in \operatorname{span}_{\mathcal{K}(\vartheta]}\{\mathrm{d} \varphi\}$ for $i=1, \ldots, k$. Thus, $\operatorname{span}_{\mathcal{K}(\vartheta]}\left\{\omega_{1}, \ldots, \omega_{k}\right\}=\operatorname{span}_{\mathcal{K}(\vartheta]}\{\mathrm{d} \varphi\}$.

Since $I_{\infty}^{p} \subseteq I_{\infty}^{p+1}$ for any $p \geq 0$, one can check the condition (5) step-by-step, increasing the value of $p$ every step. When for some $p=\bar{p}$ the condition (5) is satisfied, then it is satisfied for all $p>\bar{p}$.

Given the set of 1 -forms $\left\{\omega_{1}, \ldots, \omega_{k}\right\}$, independent over $\mathcal{K}(\vartheta]$, the basis of vector space $I_{\infty}^{s(k-1)}$ defines the basis for the largest integrable left-submodule contained in $\operatorname{span}_{\mathcal{K}(\vartheta]}\left\{\omega_{1}, \ldots, \omega_{k}\right\}$.

Lemma 2: A set of 1-forms $\left\{\omega_{1}, \ldots, \omega_{k}\right\}$ is weakly integrable if and only if the left closure of the left-submodule, generated by $\left\{\omega_{1}, \ldots, \omega_{k}\right\}$, is (strongly) integrable.

Proof: Necessity. By definitions of weak integrability and left closure, there exist functions $\varphi=\left(\varphi_{1}, \ldots, \varphi_{k}\right)^{T}$ such that $\mathrm{d} \varphi=A(\vartheta) \bar{\omega}$, where $\bar{\omega}$ is the basis of the closure of the left-submodule, generated by $\left\{\omega_{1}, \ldots, \omega_{k}\right\}$. Choose $\left\{\mathrm{d} \varphi_{1}, \ldots, \mathrm{d} \varphi_{k}\right\}$ such that for $i=1, \ldots, k$

$$
\mathrm{d} \varphi_{i} \neq a \mathrm{~d} \phi+\sum_{j=1 ; j \neq i}^{k} b_{j}(\vartheta) \mathrm{d} \varphi_{j}
$$

for any $\phi \in \mathcal{K}$ and $b_{j}(\vartheta) \in \mathcal{K}(\vartheta]$. It remains to show that one can choose $\varphi$ such that $\bar{\omega}_{i} \in \operatorname{span}_{\mathcal{K}(\vartheta]}\{\mathrm{d} \varphi\}$.

By contradiction, assume that one can not choose $\varphi$ such that $\bar{\omega}_{i} \in \operatorname{span}_{\mathcal{K}(\vartheta]}\{\mathrm{d} \varphi\}$. Then $\bar{\omega}_{k} \notin \operatorname{span}_{\mathcal{K}(\vartheta]}\{\mathrm{d} \varphi\}$ and also $\bar{\omega}_{k}^{-j} \notin \operatorname{span}_{\mathcal{K}(\vartheta]}\left\{\mathrm{d} \varphi_{1}, \ldots, \mathrm{d} \varphi_{k}\right\}$ for $j \geq 1$ and any $\varphi$. Really, if

$$
\bar{\omega}_{k}^{-j}=\sum_{i} c_{i}(\vartheta) \mathrm{d} \varphi_{i}
$$

then, since on the left-hand side of (10) everything is delayed at least $j$ times, everything that is delayed less than $j$ times on the right-hand side should cancel out. Therefore, one is able to find functions $\phi_{i}, \psi_{i} \in \mathcal{K}, i=1, \ldots, k$, such that $\mathrm{d} \varphi_{i}=\mathrm{d} \phi_{i}+\mathrm{d} \psi_{i}$ and

$$
c_{i}(\vartheta) \mathrm{d} \phi_{i} \in \operatorname{span}_{\mathcal{K}(\vartheta]}\left\{\mathrm{d} x^{-j}\right\} \quad \sum_{i} c_{i}(\vartheta) \mathrm{d} \psi_{i}=0 .
$$

Now, because of (9), $\psi_{i}=0, \phi_{i}=\varphi_{i}$ for $i=1, \ldots, k$ and thus $\delta^{j} \bar{\omega}_{k}=\delta^{j} \sum_{i} \bar{c}_{i}(\vartheta) \mathrm{d} \varphi_{i}^{+j}$ which yields $\bar{\omega}_{k}=\sum_{i} \bar{c}_{i}(\vartheta) \mathrm{d} \varphi_{i}^{+j}$. Clearly, 1-forms $\mathrm{d} \varphi_{i}^{+j}$ have to belong to $\operatorname{span}_{\mathcal{K}(\vartheta]}\{\bar{\omega}\}$, because $\mathrm{d} \varphi_{i} \in \operatorname{span}_{\mathcal{K}(\vartheta]}\{\bar{\omega}\}$. Now, one has a contradiction and therefore $\bar{\omega}_{k}^{-j} \notin \operatorname{span}_{\mathcal{K}(\vartheta]}\{\mathrm{d} \varphi\}$ for $j \geq 1$. Then, by construction $\operatorname{span}_{\mathcal{K}(\vartheta]}\left\{\mathrm{d} \varphi_{1}, \ldots, \mathrm{d} \varphi_{k}\right\} \subset \operatorname{span}_{\mathcal{K}(\vartheta]}\left\{\omega_{1}, \ldots, \omega_{k-1}\right\}$, which is impossible. Thus, the assumption that one can not choose $\varphi$ such that $\bar{\omega}_{i} \in \operatorname{span}_{\mathcal{K}(\vartheta]}\{\mathrm{d} \varphi\}$ must be wrong.
Sufficiency. Sufficiency is satisfied directly by the definitions of strong and weak integrability.

Example 5: Consider the following 1-forms

$$
\begin{aligned}
& \omega_{1}=x_{3}(t-1) \mathrm{d} x_{2}(t)+x_{2}(t) \mathrm{d} x_{3}(t-1)+x_{2}(t-1) \mathrm{d} x_{1}(t-1) \\
& \omega_{2}=x_{3}(t-2) \mathrm{d} x_{2}(t-1)+x_{2}(t-1) \mathrm{d} x_{3}(t-2) \\
& \quad+\mathrm{d} x_{1}(t)+x_{2}(t-2) \mathrm{d} x_{1}(t-2)
\end{aligned}
$$

One gets for $s(k-1)=2$ :

$$
I_{\infty}^{2}=\operatorname{span}_{\mathcal{K}}\left\{\mathrm{d} x_{1}(t), \mathrm{d} x_{1}(t-1), \mathrm{d}\left(x_{2}(t) x_{3}(t-1)\right)\right\} .
$$

When one eliminates the basis elements, which are dependent over $\mathcal{K}(\vartheta]$, one gets that the rank of $\operatorname{span}_{\mathcal{K}(\vartheta]}\left\{\mathrm{d} x_{1}(t), \mathrm{d} x_{1}(t-\right.$ $\left.1), \mathrm{d}\left(x_{2}(t) x_{3}(t-1)\right)\right\}$ is 2 . To check the condition (5), one has to check whether there exists a matrix $A(\vartheta)$ such that $\omega=A(\vartheta) \mathrm{d} \varphi$, where $\omega=\left(\omega_{1}, \omega_{2}\right)^{T}, \varphi=\left(\varphi_{1}, \varphi_{2}\right)^{T}, \varphi_{1}=$ $x_{2}(t) x_{3}(t-1), \varphi_{2}=x_{1}(t)$. In fact, $\omega=A(\vartheta) \mathrm{d} \varphi$, where the unimodular matrix $A(\vartheta)$ is defined in Example 1. Thus, the 1-forms (11) are strongly integrable.

Example 6: Consider the following 1-forms:

$$
\begin{aligned}
\omega_{1}= & \mathrm{d} x_{2}(t) \\
\omega_{2}= & x_{4}(t-1) \mathrm{d} x_{1}(t)+x_{2}(t) \mathrm{d} x_{2}(t-1)+x_{1}(t) \mathrm{d} x_{4}(t-1) \\
\omega_{3}= & x_{3}(t) x_{4}(t) \mathrm{d} x_{2}(t)+x_{2}(t) x_{4}(t) \mathrm{d} x_{3}(t) \\
& \quad+x_{3}(t-1) \mathrm{d} x_{2}(t-1)+x_{2}(t-1) \mathrm{d} x_{3}(t-1) .
\end{aligned}
$$

For $s(k-1)=2: I_{\infty}^{2}=\operatorname{span}_{\mathcal{K}}\left\{\mathrm{d} x_{2}(t), \mathrm{d}\left(x_{4}(t-\right.\right.$ 1) $\left.\left.x_{1}(t)\right), \mathrm{d} x_{2}(t-1), \mathrm{d} x_{2}(t-2), \mathrm{d}\left(x_{4}(t-2) x_{1}(t-1)\right)\right\}$. Now, $\omega_{1} \in I_{\infty}^{2}$ and $\omega_{2} \in I_{\infty}^{2}$, but $\omega_{3} \notin I_{\infty}^{2}$. Thus, 1-forms (11) are not strongly integrable, and $\operatorname{span}_{\mathcal{K}(\vartheta]}\left\{\mathrm{d} x_{2}(t), \mathrm{d}\left(x_{4}(t-\right.\right.$ 1) $\left.\left.x_{1}(t)\right)\right\}$ is the largest integrable left-submodule, contained in $\mathcal{A}=\operatorname{span}_{\mathcal{K}(\vartheta]}\left\{\omega_{1}, \omega_{2}, \omega_{3}\right\}$.

Now, one can check if 1 -forms (11) are weakly integrable. For that, one has to compute the left closure of $\mathcal{A}$ and check if it is strongly integrable. In practice, the left closure of a leftsubmodule $\mathcal{A}$ can be computed as the left-kernel of its rightkernel $\Delta$. Thus, the right-kernel of $\mathcal{A}$ is $\Delta=\operatorname{span}_{\mathcal{K}(\vartheta]}\{q(\vartheta)\}$, where $q(\vartheta)=\left(x_{1}(t) \vartheta, 0,0,-x_{4}(t)\right)^{T}$. The left-kernel of $\Delta$ is

$$
c l_{\mathcal{K}(\vartheta]}(\mathcal{A})=\operatorname{span}_{\mathcal{K}(\vartheta]}\left\{\mathrm{d} x_{2}(t), \mathrm{d} x_{3}(t), \mathrm{d}\left(x_{4}(t-1) x_{1}(t)\right)\right\} .
$$

Therefore, the 1-forms (11) are weakly integrable.

\section{INTEGRABILITY OF RIGHT-SUBMODULES}

Since the left annihilator of a right submodule is by construction closed, the integrability of a right submodule refers only to weak integrability. Consider the right-submodule

$$
\Delta=\operatorname{span}_{\mathcal{K}(\vartheta]}\left\{q_{1}(\vartheta), \ldots, q_{k}(\vartheta)\right\},
$$

where $q_{i}(\vartheta)$ are the $n \times 1$ column vectors.

Definition 7: The right-submodule $\Delta$ is said to be integrable if the left-kernel of $\Delta$ admits an exact basis.

Define a matrix $Q(\vartheta)=\left(q_{1}(\vartheta), \cdots, q_{l}(\vartheta)\right)$ and let $Q(\vartheta)=$ $Q_{0}+Q_{1} \vartheta+\cdots+Q_{s} \vartheta^{s}$ for some $s \geq 0$ and matrices $Q_{j} \in \mathcal{K}^{n \times k}, j=0, \ldots, s$. Assume, that the ranks of matrices 
$Q(\vartheta)$ and $Q_{0}$ are $k$. Consider the distributions $\Delta_{i}$ defined on $\mathbb{R}^{(i+s+1) n}$,

$\Delta_{i}:=\operatorname{span}_{\mathcal{K}}\left\{\begin{array}{cccccc}Q_{0} & \cdots & Q_{s} & 0 & \cdots & \cdots \\ 0 & \ddots & \cdots & \ddots & 0 & \cdots \\ \vdots & \ddots & \delta^{i} Q_{0} & \cdots & \delta^{i} Q_{s} & 0 \\ 0 & \cdots & & \cdots & 0 & I_{n s}\end{array}\right\}, i \geq 0$

Theorem 2: [4] The right-submodule $\Delta$ is integrable if and only if there exists an integer $\gamma$ such that, locally around some point $x^{0}(\cdot), \operatorname{dim}\left(\bar{\Delta}_{\gamma}\right)-\operatorname{dim}\left(\bar{\Delta}_{\gamma-1}\right)=k$.

The integrability of right-submodules and 1-forms are connected by the following corollary, which follows from Corollary 2 and Lemma 1.

Corollary 1: Weak integrability of 1-forms is equivalent to the integrability of its right-kernel.

To show more explicitly how the integrability of rightsubmodules and weak integrability of 1-forms are related, consider the Algorithm (1) inizialized with (4). The left-kernel of $\Delta_{i}$, defined above, is equal to $I_{\infty}^{i}$, where $I_{\infty}^{i}$ is computed with respect to the closure of a given submodule.

The next example shows, that in some cases, one can not use the results of Section IV to check the integrability of 1-forms. In that case, one has to use the results of Section III.

Example 7: Consider the 1-forms

$$
\begin{aligned}
\omega_{1}= & x_{1}(t-1) \mathrm{d} x_{1}(t)+x_{1}(t) \mathrm{d} x_{1}(t-1) \\
& -x_{3}(t) \mathrm{d} x_{2}(t-1)+\mathrm{d} x_{3}(t-1) \\
\omega_{2}= & \mathrm{d} x_{2}(t)+x_{3}(t) \mathrm{d} x_{2}(t-1) .
\end{aligned}
$$

The 1-forms $\omega=\left(\omega_{1}, \omega_{2}\right)^{T}$ can be written as

$$
\omega=\left(\begin{array}{ccc}
x_{1}(t-1)+x_{1}(t) \vartheta & -x_{3}(t) \vartheta & \vartheta \\
0 & 1+x_{3}(t) \vartheta & 0
\end{array}\right) \mathrm{d} x(t) .
$$

The right-kernel of the left-submodule $\operatorname{span}_{\mathcal{K}(\vartheta]}\left\{\omega_{1}, \omega_{2}\right\}$ is not causal (i.e. one needs forward-shifts of variables $x(t)$ to represent it), thus one can not use Theorem 2 to check the weak integrability of 1-forms (12). But, one can check by using Corollary 2 and Theorem 1, that $\operatorname{span}_{\mathcal{K}(\vartheta]}\left\{\omega_{1}, \omega_{2}\right\} \subset$ $\operatorname{span}_{\mathcal{K}(\vartheta]}\left\{\mathrm{d}\left(x_{1}(t) x_{1}(t-1)+x_{3}(t-1)\right), \mathrm{d} x_{2}(t)\right\}$ and thus, 1-forms (12) are weakly integrable.

\section{ApPlicAtions of INTEGRABILITy}

In this Section, two problems are considered, where integrability of 1-forms is used. First, it is shown that accessibility of nonlinear time-delay systems can be characterized through integrability of a certain left-submodule. Secondly, necessary and sufficient conditions are given to transform a nonlinear time-delay system into the form (17) below.

Consider the nonlinear time-delay system

$$
\dot{x}(t)=f\left(x(t-i), u(t-i) ; i=0, \ldots, d_{\max }\right),
$$

where $x(t) \in \mathbb{R}^{n}$ and $u(t) \in \mathbb{R}^{m}$. Also, assume that the function $f$ is meromorphic. To simplify the presentation, the following notation is used: $x(\cdot):=(x(t), x(t-1), \ldots)$. The notation $\varphi(x(\cdot))$ means that function $\varphi$ can depend on $x(t), \ldots, x(t-i)$ for some finite $i \geq 0$. The same notation is used for other variables.
In this section $\mathcal{K}_{u}$ denotes the field of meromorphic functions that depend on a finite number of variables from the set $\mathcal{C}=\left\{x(\cdot), u(\cdot), \ldots, u^{(k)}(\cdot) ; k \in \mathbb{N}\right\}$. Also, denote by $\mathcal{E}_{u}$ the vector space spanned by the symbols $\mathrm{d} \mathcal{C}=$ $\left\{\mathrm{d} x(\cdot), \mathrm{d} u(\cdot), \ldots, \mathrm{d} u^{(k)}(\cdot) ; k \in \mathbb{N}\right\}$ over the field $\mathcal{K}_{u}$ and $\mathcal{M}_{u}=\operatorname{span}_{\mathcal{K}_{u}(\vartheta]}\left\{\mathrm{d} x(t), \mathrm{d} u^{(k)}(t) ; k \geq 0\right\}$ is the corresponding module spanned over the ring $\mathcal{K}_{u}(\vartheta]$.

Definition 8: A 1-form $\omega \in \operatorname{span}_{\mathcal{K}_{u}(\vartheta]}\{\mathrm{d} x(t)\}$ has relative degree $r$, if $r$ is the smallest integer such that $\omega^{(r)} \notin$ $\operatorname{span}_{\mathcal{K}_{u}(\vartheta]}\{\mathrm{d} x(t)\}$. A function $\varphi \in \mathcal{K}_{u}$ is said to have relative degree $r$ if the 1-form $\mathrm{d} \varphi$ has relative degree $r$.

Define a sequence of left-submodules $\mathcal{H}_{1} \supset \mathcal{H}_{2} \supset \ldots$ of $\mathcal{M}_{u}$ as follows:

$$
\begin{aligned}
\mathcal{H}_{1} & =\operatorname{span}_{\mathcal{K}_{u}(\vartheta]}\{\mathrm{d} x(t)\} \\
\mathcal{H}_{i} & =\operatorname{span}_{\mathcal{K}_{u}(\vartheta]}\left\{\omega \in \mathcal{H}_{i-1} \mid \dot{\omega} \in \mathcal{H}_{i-1}\right\} .
\end{aligned}
$$

Since $\mathcal{H}_{1}$ has finite rank and all the left-submodules $\mathcal{H}_{i}$ are closed, sequence (14) converges (see [9]). Let $\mathcal{H}_{\infty}$ be the limit of sequence (14). By $\hat{\mathcal{H}}_{i}$ one denotes the largest integrable leftsubmodule contained in $\mathcal{H}_{i}$. A left-submodule $\mathcal{H}_{i}$ contains all the 1 -forms with relative degree $i$ or bigger. Thus, $\mathcal{H}_{\infty}$ contains all the 1 -forms which have infinite relative degree.

\section{A. Accessibility}

In this subsection the accessibility property of system (13) is characterized using the notion of autonomous element, as is done in [7] for delay-free systems, or in [18] for linear time-delay systems through the notion of torsion elements.

Definition 9: A nonzero function $\varphi \in \mathcal{K}_{u}$ is said to be an autonomous element of system (13) if there exist an integer $\nu$ and a nonzero function $F \in \mathcal{K}_{u}$ such that

$$
F\left(\varphi, \dot{\varphi}, \ldots, \varphi^{(\nu)}\right)=0 .
$$

Now, accessibility of system (13) can be defined as nonexistence of autonomous elements.

Definition 10: System (13) is said to be accessible if there does not exist any autonomous element.

Lemma 3: Function $\varphi \in \mathcal{K}_{u}$ is an autonomous element of system (13) if and only if it has infinite relative degree.

Proof: Necessity. Let $\varphi$ be an autonomous element of system (13) and assume it has finite relative degree. Then, $\operatorname{dim}\left(\operatorname{span}_{\mathcal{K}_{u}(\vartheta]}\left\{\mathrm{d} \varphi, \ldots, \mathrm{d} \varphi^{(k-1)}\right\}\right)=k$ for all $k \geq 1$. Because of (15), the last equality is not satisfied for $k=\nu+1$, which is a contradiction. Thus, $\varphi$ has infinite relative degree.

Sufficiency. Let $\varphi$ be a nonzero function with infinite relative degree. Then 1 -forms $\mathrm{d} \varphi, \ldots, \mathrm{d} \varphi^{(n)}$ are dependent over the ring $\mathcal{K}_{u}(\vartheta]$. Thus, there exist $a_{i} \in \mathcal{K}_{u}(\vartheta], i=0, \ldots, n$, where at least one of them is nonzero, such that

$$
\omega:=a_{0} \mathrm{~d} \varphi+\cdots+a_{n} \mathrm{~d} \varphi^{(n)}=0 .
$$

Then, there exists a delay differential equation as $\alpha\left(\delta, \varphi, \ldots, \varphi^{(n)}\right)=0$. By Definition 9 function $\varphi$ is an autonomous element of system (13).

Now, one can characterize accessibility of system (13) in the following way. 
Theorem 3: System (13) is accessible if and only if $\hat{\mathcal{H}}_{\infty}=\emptyset$.

Proof: Necessity. If system (13) is accessible, then by Lemma 3 there does not exist any non constant function in $\mathcal{K}_{u}$ with infinite relative degree. Therefore, there can not be any exact nonzero 1 -form in $\mathcal{H}_{\infty}$ and thus $\hat{\mathcal{H}}_{\infty}=\emptyset$ must be true.

Sufficiency. The left-submodule $\mathcal{H}_{\infty}$ contains all the 1 -forms with infinite relative degree. Since $\hat{\mathcal{H}}_{\infty}=\emptyset$, there is no non constant exact 1 -form with infinite relative degree and therefore, by Lemma 3, there is no autonomous element.

\section{B. Normal form}

In this subsection, one considers the possibility of transforming (13), with one single input $(m=1)$, into the form

$$
\begin{aligned}
& \dot{z}^{1}(t)=f_{1}\left(z^{1}(\cdot), u(\cdot)\right) \\
& \dot{z}^{2}(t)=f_{2}\left(z^{1}(\cdot), z^{2}(\cdot)\right),
\end{aligned}
$$

where the dynamics corresponding to $z^{1}(t)$ is accessible, by a state transformation $z(t)=\varphi(x(\cdot))$ and a regular static feedback $u(t)=\alpha(x(\cdot), v(\cdot))$.

To solve the above mentioned problem, first, we define invariant and controlled invariant left-submodules. For that, consider a left-submodule $\mathcal{A}=\operatorname{span}_{\mathcal{K}_{u}(\vartheta]}\left\{\omega_{1}, \ldots, \omega_{k}\right\}$ and let $\dot{\mathcal{A}}=\operatorname{span}_{\mathcal{K}_{u}(\vartheta]}\left\{\dot{\omega}_{1}, \ldots, \dot{\omega}_{k}\right\}$.

Definition 11: A left-submodule $\mathcal{A} \subseteq \operatorname{span}_{\mathcal{K}_{u}(\vartheta]}\{\mathrm{d} x(t)\}$ is said to be invariant if $\dot{\mathcal{A}} \subseteq \operatorname{cl}_{\mathcal{K}_{u}(\vartheta]}(\mathcal{A})+\operatorname{span}_{\mathcal{K}_{u}(\vartheta]}\{\mathrm{d} u(t)\}$.

Definition 12: A left-submodule $\mathcal{A} \subseteq \operatorname{span}_{\mathcal{K}_{u}(\vartheta]}\{\mathrm{d} x(t)\}$ is said to be controlled invariant if there exists a regular feedback $u(t)=\alpha(x(\cdot), v(\cdot))$ such that $\dot{\mathcal{A}} \subseteq c_{\mathcal{K}_{u}(\vartheta]}(\mathcal{A})+$ $\operatorname{span}_{\mathcal{K}_{u}(\vartheta]}\{\mathrm{d} v(t)\}$.

Theorem 4: System (13), where $m=1$, can be transformed into the form (17), where $\operatorname{dim} z^{1}(t)=k$, by a state transformation $z(t)=\varphi(x(\cdot))$ and a regular static feedback $u(t)=\alpha(x(\cdot), v(\cdot))$ if and only if

(i) $\operatorname{rank} \hat{\mathcal{H}}_{2} \geq n-k$

(ii) there exists a weakly integrable controlled invariant leftsubmodule $\mathcal{A}$ with rank $k$ such that $\mathcal{A} \cap \hat{\mathcal{H}}_{\infty}=\emptyset$ and $\mathcal{A}$ contains $\mathcal{H}_{1} / \hat{\mathcal{H}}_{2}$.

Proof: Necessity. Since $\operatorname{dim} z^{1}(t)=k$, then $\operatorname{dim} z^{2}(t)=$ $n-k$. Because the first order time derivatives of $z^{2}(t)$ do not depend on the input variable, one gets that $\mathrm{d} z_{j}^{2}(t)=$ $\mathrm{d} \varphi_{j}^{2}(x(\cdot)) \in \hat{\mathcal{H}}_{2}, j=1, \ldots, n-k$. Therefore, since $z_{j}^{2}$, $j=1, \ldots, n-k$ are independent, condition $(i)$ is satisfied.

Let $\mathcal{A}=\operatorname{span}_{\mathcal{K}_{u}(\vartheta]}\left\{\mathrm{d} z^{1}\right\}$. Clearly, this left-submodule satisfies the condition $(i i)$ of Theorem 4.

Sufficiency. Because $\mathcal{A}$ is weakly integrable one has $c l_{\mathcal{K}_{u}(\vartheta]}(\mathcal{A})=\operatorname{span}_{\mathcal{K}_{u}(\vartheta]}\left\{\mathrm{d} \varphi_{1}, \ldots, \mathrm{d} \varphi_{k}\right\}$. Define $z_{i}^{1}=\varphi_{i}$, $i=1, \ldots, k$. Since $\mathcal{A}$ contains $\mathcal{H}_{1} / \hat{\mathcal{H}}_{2}$ and rank $\hat{\mathcal{H}}_{2} \geq n-k$, one can find $z^{2}=\varphi^{2}(x(\cdot))$ such that $\mathrm{d} z^{2} \in \mathcal{H}_{2}$ and $z(t)=$ $\left(z^{1}, z^{2}\right)^{T}$ is a state transformation [19]. Because $\mathcal{A}$ is controlled invariant, there exists a feedback $u(t)=\alpha(x(\cdot), v(\cdot))$ which makes $\mathcal{A}$ invariant. Finally, condition $\mathcal{A} \cap \hat{\mathcal{H}}_{\infty}=\emptyset$ guarantees accessibility of $z^{1}$.

\section{CONCLUSION}

The integrability of 1-forms, which plays an important role in the analysis of time-delay systems, was characterized. Necessary and sufficient conditions were given to check if a set of 1-forms is strongly (weakly) integrable, together with two algorithms to compute the largest integrable left-submodule, which is contained in the (closure of) left-submodule generated by the given 1 -forms. It was also shown that accessibility of nonlinear time-delay systems can be characterized through integrability of certain left-submodule.

\section{REFERENCES}

[1] J. P. Richard, Time-delay systems: an overview of some recent advances and open problems, Automatica, V.39,10, pp.1667-1694, 2003

[2] L. A. Márquez-Martínez and C. H. Moog, New insights on the analysis of nonlinear time-delay systems: Application to the triangular equivalence, Systems \& Control Letters, V.56, pp.133-140, 2007

[3] G. Conte and A. M. Perdon, The disturbance decoupling problem for systems over a ring, SIAM Journal of Control and Optimization, V.33,3, pp.750-764, 1995

[4] C. Califano and L. A. Márquez-Martínez and C. H. Moog, Extended Lie brackets for nonlinear time-delay systems, IEEE Transactions on Automatic Control, V.56, 9, pp.2213-2218, 2011

[5] C. H. Moog and R. Castro-Linares and M. Velasco-Villa and L. A Márquez-Martínez, The disturbance decoupling for time-delay nonlinear systems, IEEE Transactions on Automatic Control, V.45, 2, pp.305-309, 2000

[6] A. Isidori, Nonlinear Control Systems, Springer, London, 1995

[7] G. Conte and C. H. Moog and A. M. Perdon, Algebraic Methods for Nonlinear Control Systems. Theory and Applications. , Springer, London, 2007

[8] H. Nijmeijer and A. van der Schaft, Nonlinear Dynamical Control Systems, Springer, New York, 1990

[9] X. Xia and L. A. Márquez-Martínez and P. Zagalak and C. H. Moog, Analysis of nonlinear time-delay systems using modules over noncommutative rings, Automatica, V.38, 9, pp.1549-1555, 2002

[10] A. Germani and C. Manes and P. Pepe, Linearization and decoupling of nonlinear delay systems, Proceedings of the American Control Conference, Philadelphia, USA, 1998, pp.1948-1952

[11] T. Oguchi and A. Watanabe and T. Nakamizo, Input-output linearization of retarded non-linear systems by using an extension of Lie derivative, International Journal of Control, V.75, 8, pp.582-590, 2002

[12] L. A. Márquez-Martínez, Note sur l'accessibilité des systèmes non linéaires à retards, Comptes Rendus de l'Académie des Sciences, V.329, pp.545-550, 1999

[13] G. Zheng and J. -P. Barbot and D. Boutat, Identification of the delay parameter for nonlinear time-delay systems with unknown inputs, Automatica, V.49, 6, pp.1755-1760, 2013

[14] Y. Choquet-Bruhat and C. DeWitt-Morette and M. Dillard-Bleick, Analysis, Manifolds and Physics, Part I: Basics, North-Holland, Amsterdam, 1989

[15] P. M. Cohn, Free Rings and Their Relations, Academic Press, London, 1985

[16] S. J. Li and C. H. Moog and C. Califano, Characterization of accessibility for a class of nonlinear time-delay systems, Proceedings of the 50th IEEE Conference on Decision and Control, Orlando, Florida, 2011, pp.1068-1073

[17] A. Kaldmäe and C. H. Moog and C. Califano, Notion of integrable one-forms for nonlinear time-delay systems, 1st IFAC Conference on Modelling, Identification and Control of Nonlinear Systems, St. Petersburg, Russia, 2015, pp.910-915

[18] M. Fliess and H. Mounier, Controllability and observability of linear delay systems: an algebraic approach, ESAIM: Control, Optimization \& Calculus of Variations, V.3, pp.301-314, 1998

[19] C. Califano and C. H. Moog, Coordinates transformations in nonlinear time-delay systems, 53rd IEEE Conference on Decision and Control, Los Angeles, USA, 2014, pp.475-480 